\title{
Stay on target
}

\section{Applying a rigorous chemical biology approach to the selection and validation of clinical targets will increase the success of drug discovery initiatives.}

The drug discovery engine, largely driven by the pharmaceutical industry, is in crisis (Nat. Chem. Biol. 7, 335-339, 2011), and in response, the landscape of this essential research arena is changing at an alarming pace. These changes include major losses in revenue from the expiration of patents that necessitate new funding models for research and development (for example, publicprivate partnerships; Nat. Chem. Biol. 9, 3-6, 2013) and coincide with unsustainably high drug-failure rates. Although flux in the drug discovery industry is certainly not without precedent, at Nature Chemical Biology we believe the current crisis originates, in part, from a lack of mechanistic rigor in the selection and validation of targets. Thus, we propose that chemical biologists should view this crisis as both a challenge to and an opportunity for the field to claim its rightful position as an essential component of every drug discovery initiative.

In a prior issue (http://www.nature.com/ nchembio/focus/chemicalprobes/index. $\mathrm{html} /$ ), we presented a collection of articles focused on chemical probes, the oft-used tools that provide the most obvious connection between chemical biology and drug discovery. In this issue, we provide a complementary focus, exploring how chemical biology can add necessary rigor to the process of target selection and validation, whether the target is a protein, another biomolecule or even a phenotype. We present a collection of articles that tackle the challenges in the discovery and validation of new targets for clinical purposes, the application of chemical probes to understand the relationship between targets and biology and the expanding assortment of chemical tools and techniques that can be used to perturb targets that have been considered chemically intractable.

Bunnage et al. (p. 195) begin the issue with a Commentary that outlines a four-pillared framework for validating a target for clinical purposes, highlighting the benefits of building each pillar through a rigorous chemical biology approach. These pillars include establishing that the compound penetrates to the site of action, engages the target and affects the activity of the target as well as showing that the altered activity has desirable phenotypic consequences. A Commentary by Simon et al. (p. 200) focuses on one of these pillars-target engagement—and emphasizes the often-overlooked importance of assessing target engagement in a specific biological context before making any conclusions about phenotypes derived from the application of a chemical probe or drug designed to hit that target. Simon et al. additionally provide an overview of established and emerging methodologies to quantify target engagement in model systems and humans.

In addition to validating known targets and understanding the relationship between these targets and biological outcomes, chemical biology can provide access to new targets and establish unanticipated connections between targets and pathways. Advances in synthetic chemistry enrich the molecular libraries that chemical biologists rely on. In their Commentary, Kesavan and Marcaurelle (p. 210) highlight some recent advances in synthetic chemistry methodologies and in their application toward expanding target space. In a complementary Review article, Schenone et al. (p. 232) examine biochemical, genetic and computational approaches and their respective methodologies that allow scientists to identify the targets of compounds with interesting or desirable bioactivity. The authors emphasize that these approaches share an unbiased foundation, allowing scientists to benefit from serendipity in their research and generate unexpected hypotheses.

Targets are not limited to biomolecules; they can also be phenotypes. Indeed, phenotypic screens, largely abandoned with the rise of targeted screens, are re-emerging as a valuable approach to discover new targets. In her Commentary, Eggert (p. 206) outlines the fundamentals of running a successful phenotypic screen, emphasizing the importance of selecting a well-defined phenotype and applying rigorous and wellcontrolled assays in these screens. Eggert further defines criteria for maximizing the success of these screens, from assay design to hit validation and ultimately identification of the molecular target (or targets) responsible for the phenotype. In a parallel Perspective, Roemer and Boone (p. 222) outline a multidisciplinary target-identification strategy that can be used in a whole-cell or phenotypic context for the discovery of antimicrobials. They also emphasize the value of systems biology in the discovery of synthetic-lethal interactions that can be exploited to maximize the number of viable antimicrobial targets with the potential to have a clinical impact.

Taking this special issue as a whole, two themes emerge that particularly resonate with us. First, chemical probes and drugs are fundamentally distinct entities. For example, multiple authors emphasize that when it comes to in-cell selectivity between on- and off-target activity, chemical probes should be held to a higher standard than drugs, as clinical responses may in fact improve from off-target activity (via polypharmacology), whereas the interpretation of biological responses to chemical probes requires the deconvolution of outcomes associated with on- and off-target activities. Second, multidisciplinary approaches to quantifying target engagement are essential in the application of both chemical probes and drugs and should be performed independently for every biological system where a compound is applied.

These emerging ideas will affect editorial decisions on papers submitted to Nature Chemical Biology: as with the Commentary by Frye on chemical probes (Nat. Chem. Biol. 6, 159-161, 2010), the Commentary by Eggert (p. 206), together with feedback we receive from other experts, will form the foundation for the editorial criteria we apply to manuscripts reporting phenotypic screens. In the future, we will emphasize proximal and context-specific assays for targets, including the prioritization of manuscripts providing evidence that the target of interest has been engaged in the model system (or systems) of choice. Further, we seek submissions that provide new and validated methodologies that allow the identification of targets for interesting bioactive compounds, reveal polypharmacology or off-target activities or provide new approaches for the assessment and quantification of target engagement in a biological context.

We are delighted to bring together this collection of target-centric articles. On the basis of the opinions articulated in these pieces and our own experiences, we posit that the incorporation of a rigorous chemical biology approach to all drug discovery initiatives, ideally in tandem with the methodologies of other disciplines, will improve success rates in these programs and provide meaningful biological insight and tools even in cases where targets are not validated for clinical applications. 